SANDIA REPORT

SAND98-0562 - UC-706

Unlimited Release

Printed March 1998

\title{
An Advanced Unmanned Vehicle for Remote Applications
}

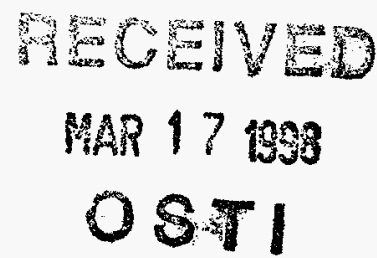

J. Bryan Pletta, John Sackos

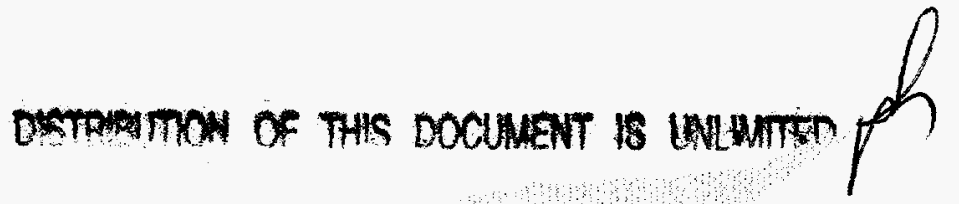

Prepared by

Sandia National Laboratories

Albuquerque, New Mexico 87185 and Livermore, California 94550

Sandia is a multiprogram laboratory operated by Sandia Corporation, a Lockheed Martin Company, for the United States Department of Energy under Contract DE-AC04-94AL85000.

Approved for public release; further dissenitation unlimited.

\section{T. Sandia National laboratories}

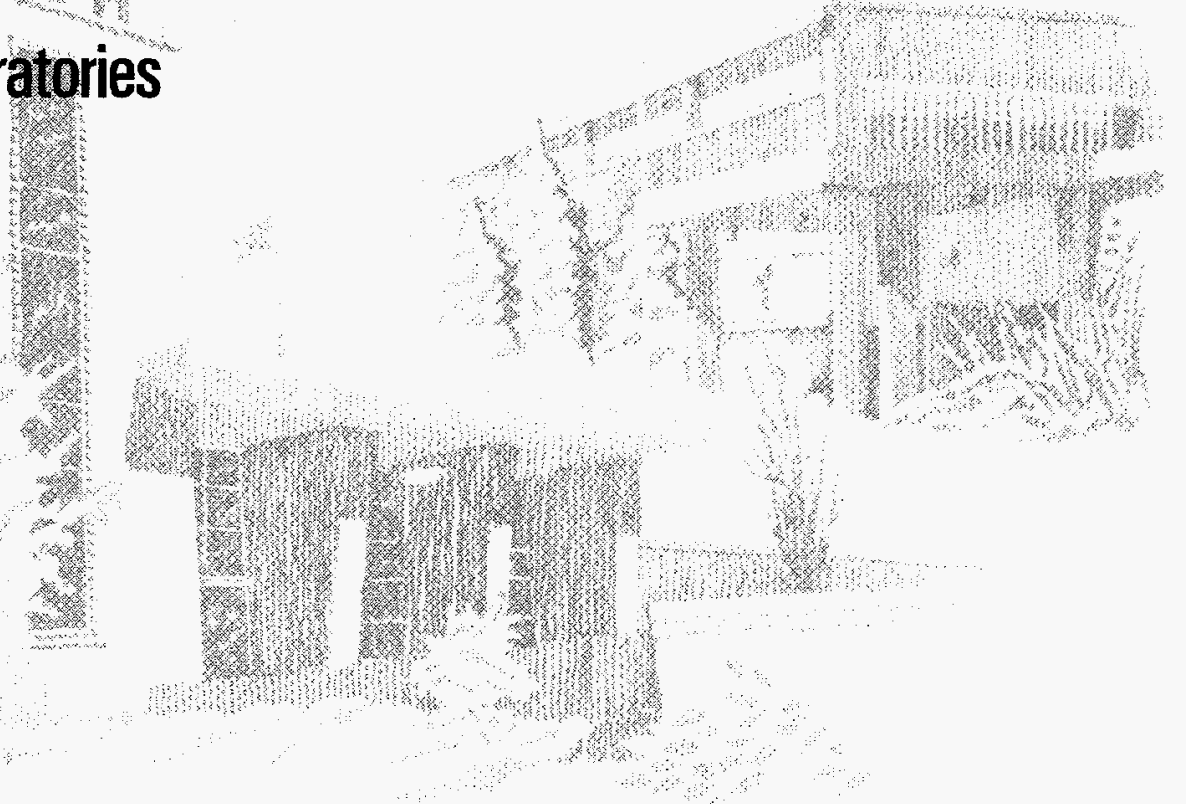


Issued by Sandia National Laboratories, operated for the United States Department of Energy by Sandia Corporation.

NOTICE: This report was prepared as an account of work sponsored by an agency of the United States Government. Neither the United States Government nor any agency thereof, nor any of their employees, nor any of their contractors, subcontractors, or their employees, makes any warranty, express or implied, or assumes any legal liability or responsibility for the accuracy, completeness, or usefulness of any information, apparatus, product, or process disclosed, or represents that its use would not infringe privately owned rights. Reference herein to any specific commercial product, process, or service by trade name, trademark, manufacturer, or otherwise, does not necessarily constitute or imply its endorsement, recommendation, or favoring by the United States Government, any agency thereof, or any of their contractors or subcontractors. The views and opinions expressed herein do not necessarily state or reflect those of the United States Government, any agency thereof, or any of their contractors.

Printed in the United States of America. This report has been reproduced directly from the best available copy.

Available to DOE and DOE contractors from

Office of Scientific and Technical Information

P.O. Box 62

Oak Ridge, TN 37831

Prices available from (615) 576-8401, FTS 626-8401

Available to the public from

National Technical Information Service

U.S. Department of Commerce

5285 Port Royal Rd

Springfield, VA 22161

NTIS price codes

Printed copy: A03

Microfiche copy: A01

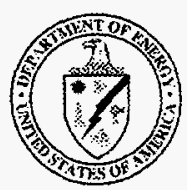




\section{DISCLAIMER}

Portions of this document may be illegible electronic image products. Images are produced from the best available original document. 
SAND98-0562

Unlimited Release

Printed March 1998

Distribution
Category UC-706

\title{
An Advanced Unmanned Vehicle for Remote Applications
}

\author{
J. Bryan Pletta \\ Mobile Robotics Department \\ John Sackos \\ Advanced Systems Department \\ Sandia National Laboratories \\ P.O. Box 5800 \\ Albuquerque, NM 87185-1125
}

\begin{abstract}
An autonomous mobile robotic capability is critical to developing remote work applications for hazardous environments. A few potential applications include humanitarian demining and ordnance neutralization, extraterrestrial science exploration, and hazardous waste cleanup. The ability of the remote platform to sense and maneuver within its environment is a basic technology requirement which is currently lacking. This enabling technology will open the door for force multiplication and cost effective solutions to remote operations.

The ultimate goal of this work is to develop a mobile robotic platform that can identify and avoid local obstacles as it traverses from its current location to a specified destination. This goal directed autonomous navigation scheme uses the Global Positioning System (GPS) to identify the robot's current coordinates in space and neural network processing of LADAR range images for local obstacle detection and avoidance.
\end{abstract}


The initial year funding provided by this LDRD project has developed a small exterior mobile robotic development platform and a fieldable version of Sandia's Scannerless Range Imager (SRI) system. The robotic testbed platform is based on the Surveillance And Reconnaissance Ground Equipment (SARGE) robotic vehicle design recently developed for the US DoD. Contingent upon follow-on funding, future enhancements will develop neural network processing of the range map data to traverse unstructured exterior terrain while avoiding obstacles. The SRI will provide real-time range images to a neural network for autonomous guidance. Neural network processing of the range map data will allow real-time operation on a Pentium based embedded processor board. 


\section{INTRODUCTION}

An autonomous mobile robotic capability is critical to developing remote work applications for hazardous environments. A few potential applications include humanitarian demining and ordnance neutralization, extra terrestrial science exploration, and hazardous waste cleanup. The ability of the remote platform to sense and maneuver within its environment is a basic technology requirement which is currently lacking. This enabling technology will open the door for force multiplication and cost effective solutions to remote operations.

Autonomous robotic vehicles will likely aid future warfighters in achieving mission objectives. A few examples of where robotics might increase land operation efficiency and safety are in reconnaissance, surveillance, building entry, target detection, sentry and patrol, building or terrain mapping, explosives neutralization, and detection of booby traps and weapon cashes. However, in order to be an effective aid to a warfighter, a tactical mobile robot must be very portable and have a high degree of autonomous mobility. To achieve this, the robot must have a reliable and accurate perception capability, which is particularly important when operating in unknown and/or unstructured environments. Because imaging optical radar offers one of the most reliable means for acquiring 3D terrain information, it satisfies most of the fundamental mobile robotic vision requirements. However, the capability of this type of perception sensor goes far beyond that of only serving the vision needs of the robot. Imaging optical radar is particularly well suited for sensing and recording the topography of all objects in a viewed scene, either for rapid model or map building, or for object (target) detection and recognition. As such, this sensor offers an opportunity to satisfy a number of mission critical robotic needs in a single sensor package.

This Lab Directed Research and Development (LDRD) project has provided support to develop a mobile robotic platform which will be used for future research and development of autonomous navigation techniques. The initial year funding provided by this LDRD project has developed a small exterior mobile robotic development platform and a fieldable version of Sandia's Scannerless Range Imager (SRI) system. The SRI is a solid state variant of Laser Radar (LADAR) technology that provides a three dimensional range image of the scene of interest. The robotic testbed platform is based on the Surveillance And Reconnaissance Ground Equipment (SARGE) robotic vehicle design recently developed for the US DoD. We have designated this upgraded robotic platform the SARGE II robotic vehicle. The SRI design was customized for parameters unique to the ground vehicle navigation application to provide range images in exterior daylight conditions over a wide field of view.

The ultimate goal of this work is to develop a mobile robotic platform that can identify and avoid local obstacles as it traverses from its current location to a specified destination. This goal directed autonomous navigation scheme will use the Global Positioning System (GPS) to identify the robot's current coordinates in space and neural network processing of LADAR range images for local obstacle detection and avoidance. 
Contingent upon follow-on funding, future enhancements will develop neural network processing of the real-time range images provided by the SRI to traverse unstructured exterior terrain while avoiding obstacles.

\section{THE SARGE ROBOTIC VEHICLE TESTBED}

The SARGE system was originally designed by Sandia Labs for the Unmanned Ground Vehicle/Systems Joint Program Office (UGV/S-JPO) as a teleoperated robot for battlefield surveillance applications. A total of eight systems were built and are being used for Early User Appraisal to aid the U.S. Army and Marines in developing tactics and doctrine for using robotic ground vehicles in tactical surveillance and reconnaissance missions. This exercise will lay the foundations for specifying requirements for the next generation Tactical Unmanned Ground Vehicle (TUGV), which is planned for inclusion in the DoD inventory. This makes the SARGE robot a particularly attractive research platform for developing new technology for military applications, since any improvements will easily transition to a fielded system.

The original SARGE system was designed for simple teleoperation and did not posses the computing power to support autonomous navigation or vision processing. That computer system was based on PC compatible industrial computer boards resident on a STD-32 backplane. The STD-32 bus specification is a high performance industrial computer bus that allows 32 bit data transfers over the backplane at rates of up to 32 Mbytes/sec and was chosen to allow a migration to support more complex requirements.

The Ziatech ZT-8901 single board computer was chosen as the SARGE main processor because of its low power consumption and extensive I/O capabilities. This board features a $16 \mathrm{MHz}$ NEC V53, which is an enhanced version of Intel's 80286 microprocessor and contains an 80287 numeric data coprocessor, 1 Mbyte static RAM, 512 Kbytes of Flash PROM, three serial ports, 48 lines of digital $\mathrm{I} / \mathrm{O}$, and various other peripherals. Additional boards are added to the system for analog $\mathrm{L} / \mathrm{O}$, quadrature decoding, and additional serial ports.

A photograph of the SARGE II robot developed under this LDRD is shown in Figure 1. The original SARGE computer architecture was modified on SARGE II to maintain the vehicle control and interface functions in the original design while extending the capability for autonomous control and vision processing on a more powerful computer. The computing system on SARGE II was upgraded to a multiprocessor design using Ziatech's STAR BIOS, which allows multiple DOS CPU's to reside on a single backplane and share common resources and data. The vehicle control processor and interface remained functionally identical to the original SARGE design, while the autonomous and high level functions were implemented on a ZT-8905 which features a $166 \mathrm{MHz}$ Pentium computer. 


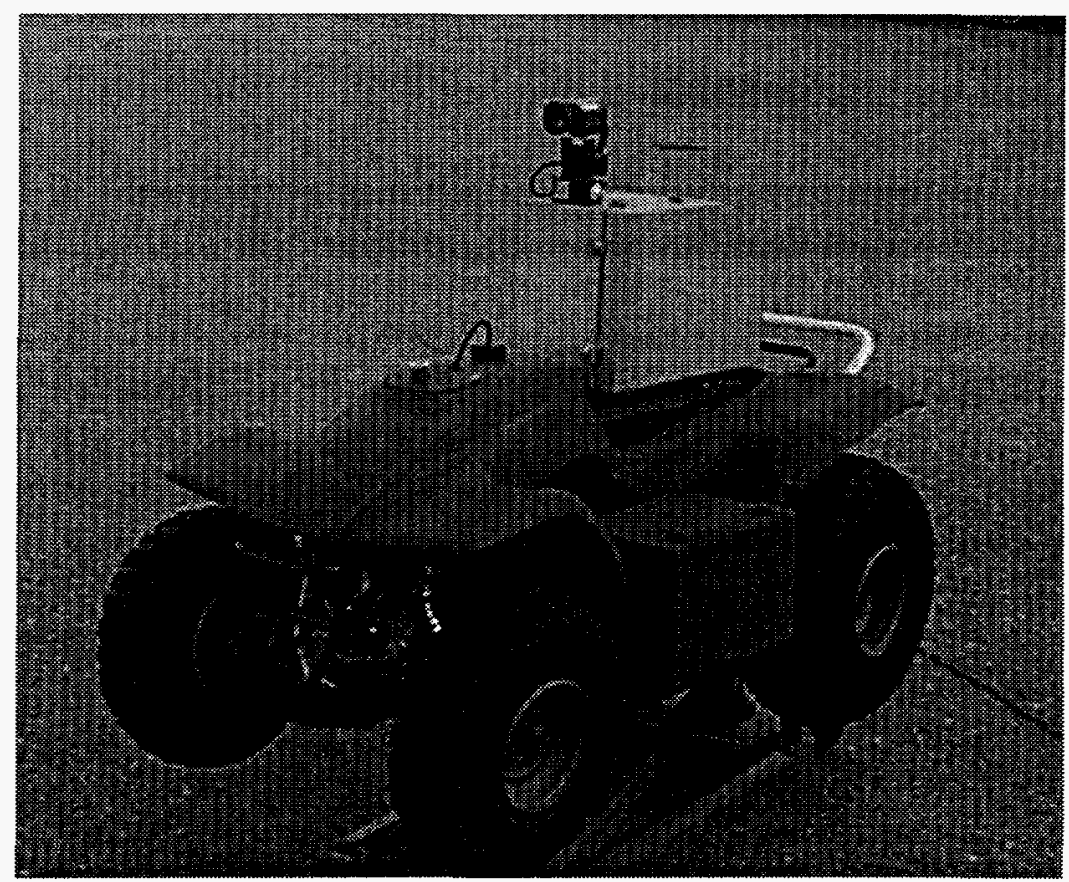

Figure 1. SARGE II Robotic Development Platform

The control system architecture was modified so that the two processors share responsibility for control. The vehicle control data structure is set in common memory by the ZT- 8905 while the ZT-8901 provides all vehicle status information as well as providing closed loop control of all actuators.

Vehicle position is determined by fusing dead reckoning and GPS data. Dead reckoning is a method of position estimation that projects the previous position estimate forward based on an incremental distance traveled at the current heading. This technique is relatively accurate over short distances but drifts as a function of distance traveled. We have typically been able to achieve approximately $2 \%$ dead reckoning accuracy in past robotics projects. In order to bound this error, a global position update is provided by an Ashtech G12 GPS receiver. The Ashtech GPS receiver is capable of providing real time position accuracy of better than $1 \mathrm{~m} \mathrm{CEP}$ when configured in a differential system.

\section{SCANNERLESS RANGE IMAGER (SRI) FOR SARGE}

Currently developed exterior autonomous vehicles navigate with either stereo TV images or scanner-based LADARs. Shadows and variations in lighting make it difficult for stereo vision based systems to reliably recognize terrain features. Passive vision based systems also typically consume significant computing resources which limit realtime performance. Non-Sandia LADAR technology exists that eliminates problems with variable lighting conditions but imposes a 1.5 second limit on data/scene updates and a 3-5 mile per hour top speed. However, Sandia's LADAR operates without a mechanical scanner, providing an update rate orders of magnitude faster than competitors and physically much more robust. 
A custom version of the Sandia Scannerless Range Imager (SRI) was fabricated for integration with the SARGE test bed. This sensor is capable of daylight operation and has a range of 50 feet with 45 degree field of view and 2 inch range resolution.

\section{BACKGROUND}

This project built on the research and exploratory development sponsored by both the DOE and DoD. For the last five year's, under a joint DoD/DOE Conventional Munitions Technology Development Program, a small group of researchers at Sandia National Laboratories have been working on a unique type of imaging optical radar technology, generally referred to as Scannerless Range Imaging (SRI) technology. Under this program, the technology was optimized for low cost missile seeker guidance applications, and had been matured to a state in which inexpensive field portable hardware was undergoing field testing at several of the service laboratories. Under this LDRD we further developed the SRI technology for application to a mobile robotic vehicle. This development miniaturized the instrument architecture, and resulted in a very capable, state-of-the-art optimized imaging radar instrument that is well suited for integration onto a small mobile robot.

\section{SENSOR COMPARISONS}

Although no one sensor technology is likely to satisfy all robot perception needs in all battlefield environments, an active imaging optical sensor does provide many significant advantages over other competing sensor technologies, such as radio frequency (RF), millimeter wave, passive infrared (IR), passive video, and stereo vision. In general, sensors that operate at optical frequencies have inherently high resolution and are particularly well suited for high fidelity, image-quality data collection. The availability of high quality range imagery also makes the development and implementation of robust obstacle detection and discrimination algorithms far easier than those derived from other perception sensor technologies. Range and reflectance imagery derived from an active system is also far less affected by ambient conditions such as changes in solar illumination and background temperatures, effects which can cause large changes in target-to-background contrast in many types of passive sensors. Operation at night, or in dark or dimly lit indoor conditions (environments which are very likely be encountered in the urban battlespace environment) can also render many passive video based vision systems useless. Because of the environmentally invariant geometric signature of a target, active range-imaging sensors also make automatic target recognition easier and countermeasures much more difficult.

However, optical range imaging sensors have some drawbacks. They do not see through solid objects or through conditions such as dense fog, smoke, or vegetation. Although some degree of sensor performance can be achieved in these environments, sensor 
performance can be significantly degraded, as compared to operation in a good visibility environment.

Most current imaging optical radar systems use problematic mechanical scanning subsystems that typically involve some type of moving mirror or optical assembly. A scannerless laser radar system has potentially significant reliability, cost, and size advantages over other types of Laser Detection and Ranging (LADAR) systems that employ mechanical scanning techniques. Although some types of scannerless laser radar concepts have been built in the past, all required either multiple and/or gated receivers, and none provided very good range resolution because of gate bandwidth limitations. Because of pixel density limitations, the spatial resolution of low cost $(<\$ 50 \mathrm{~K})$ LADARs are typically not very good. The need for some type of beam scanning is also one of the principal problems with all current scanned LADAR sensors systems. By providing pixel range determination using the combination of an inexpensive focal panel array detector and an inexpensive commercially available control computer, Sandia's Scannerless Range Imager (SRI) system reduces cost, extends performance, and eliminates the reliability deficiencies of the more bulky, and power intensive, conventionally scanned range imaging systems.

\section{TECHNOLOGY DESCRIPTION}

Sandia's Scannerless Range Imager is a compact, low-cost, high-resolution, high-frame rate, scannerless, range-imaging optical radar. The SRI technology allows fast formation of a range image over a large field of view (object plane) without the use of any type of beam steering or scanning subsystem. The SRI is a floodlight-illuminated, total field-of-view (staring) LADAR system that uses an intensity-modulated lightsource transmitter along with an image-intensified charge coupled device (ICCD) video camera receiver. Depending on the desired operating range of the system, either a laser or an array of eye-safe light-emitting diodes (LEDs) may be used as the system transmitter (light illuminator/modulator). Continuous wave (cw), pulsed, quasi-cw semiconductor lasers, and diode or flash lamp pumped crystal lasers have all been demonstrated to be suitable light sources for the SRI technology. Both semiconductor lasers and LEDs are low cost and either are or can be made relatively eye safe through spectral wavelength selection and/or spatial distribution of the light source (a technique that is not practical for use with beam-scanned LADAR systems).

The SRI technology is based on conventional cw phase detection electromagnetic radar theory. Real-time numerical extraction of pixel range measurements from the amplitude-modulated scene illumination is made possible by predetection mixing of the return signal within an image intensifier and subsequent extraction of the demodulated phase signal using a microprocessor-based digital-signal processor. The light source and the image intensifier are synchronously modulated to produce an amplitude variation in the scene illumination and a sinusoidal modulation in the sensitivity of the camera based receiver. Because the two elements are modulated at the same frequency, a mixing occurs in the image intensifier. The mixing preserves the phase difference between the 
received laser light that is reflected back from objects in the viewed scene and the modulated image intensifier gain, which acts as a type of injected local oscillator. The mixing and detection of phase occurs simultaneously for each pixel across the entire imaged scene. Because of this predetection mixing, the phase information can be collected and extracted using an integrating detection element such as the charge coupled device (CCD).

The SRI technology can most easily operate within the visible or near-infrared regions of the electromagnetic spectrum. The bounds of available image intensifier photocathode device sensitivity and the availability of compatible light illuminators define this region. As a result of the electromagnetic wavelength of operation, the SRI has inherently high spatial resolution and is particularly well suited for high fidelity, image-quality data collection. In addition to range imagery, an important feature or consequence of the SRI system is the automatic generation of pixel-registered, actively illuminated photographic imagery. This range imaging scheme also minimizes the need for stringent uniformity requirements on the illuminating light source, thereby further reducing transmitter cost and improving system reliability.

To date, Sandia has produced several versions of the SRI sensor system using commercially available digital video cameras from NEC, Dalsa, Kodak, SMD, and Cidtec. The camera is connected to a host personal computer (PC) via commercial frame grabber electronics. The PC provides real-time control of the system and display of the collection reflectance and processed range images. The SRI system is operated through a graphical user software interface program that serves to both control the SRI system and implement the various processing algorithms required to produce range images from collected reflectance images. Convenient data value interrogation, manipulation, and display of the reflectance and range images are achieved with this user-friendly graphical interface and control software program. The operating environment software is written in very efficient $C$ language and provides a dualwindow display with pop-up, push-button menus that respond to point-and-click mouse operations. The user interface software also controls the storage, loading, and manipulation of all image data associated with the system and also includes embedded online documentation that allows easy access to many of the menu-driven control features of the system.

\section{PERFORMANCE ACCOMPLISHMENTS}

In terms of LADAR technology, the Sandia system is presently the only laser radar system in the world that forms an entire range image in a floodlight mode (total scene illuminated all at once). With larger laser sources, we have demonstrated very high resolution (one inch range resolution) in both day and night conditions out to distances of over one kilometer. The technology also offers an opportunity to significantly increase the frame rate of current sub-Hertz frame rate LADAR systems to video rates (30 Hz and greater). 


\section{SENSOR ARCHITECTURE}

The architecture of the SARGE II SRI sensor consists of the following transmitter and receiver assembly. The receiver assembly consists of a camera lens and bandpass filter, an image intensifier, high-speed control electronics, a digital camera, and a computer with both digital and analog I/O and an Ethernet port. The transmitter assembly consists of a stacked semiconductor laser diode array, and drive electronics. These components are shown in Figures 2 and 3, and the hand shown in the pictures illustrates the overall physical size of the instrument. A more detailed discussion of these components is provided in separate sections below.

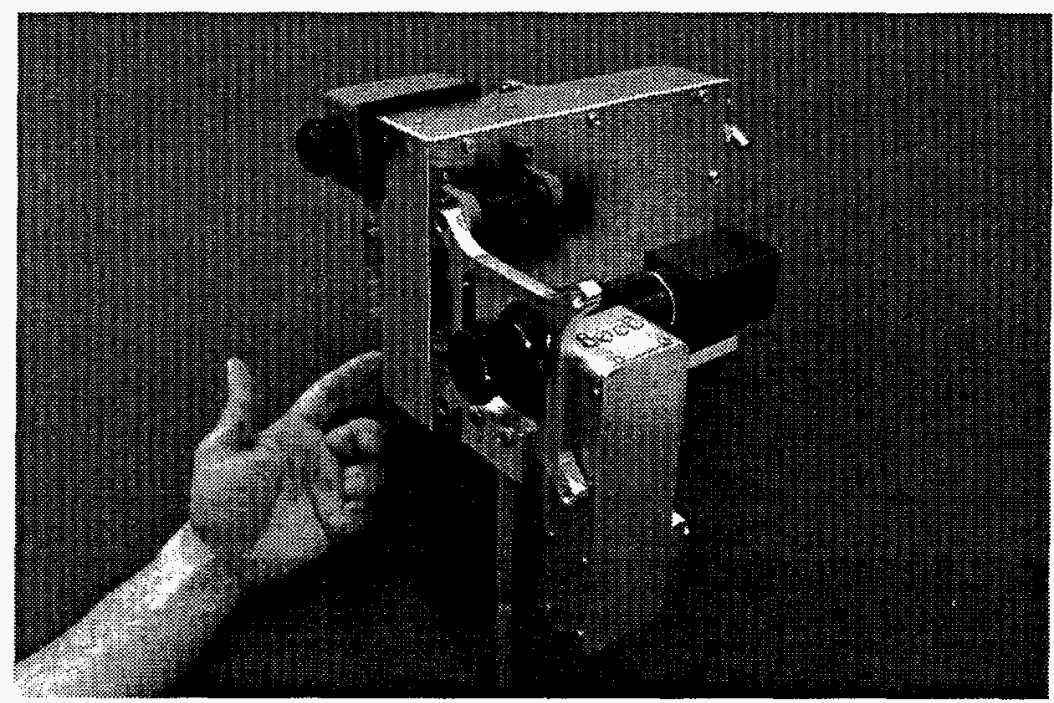

Figure 2. Scannerless Range Imager Laser Transmitter and Receiver

\section{Camera Lens and Bandpass Filter}

A conventional miniature video $\mathrm{C}$-mount camera lens is used to image the scene onto the photosensitive area of the image intensifier's photocathode. An $8 \mathrm{~mm}$ focal length $f$ 1.2 lens provides the sensor with a 45 - 50 degree full field-of-view (FOV). A $25 \mathrm{~mm}$, $10 \mathrm{~nm}$ FWHM band pass optical filter inserted into the optical path of the lens assembly provides adequate rejection of background radiation from solar and all other unwanted light sources that may be illuminating the scene. The filter is centered on the operating emission line of the laser. Spectral tolerance is incorporated in this design to accommodate for both the variation in wavelength emission due to temperature, and for the angular sensitivity of the filter to the desired broad field viewing angle of the sensor. 


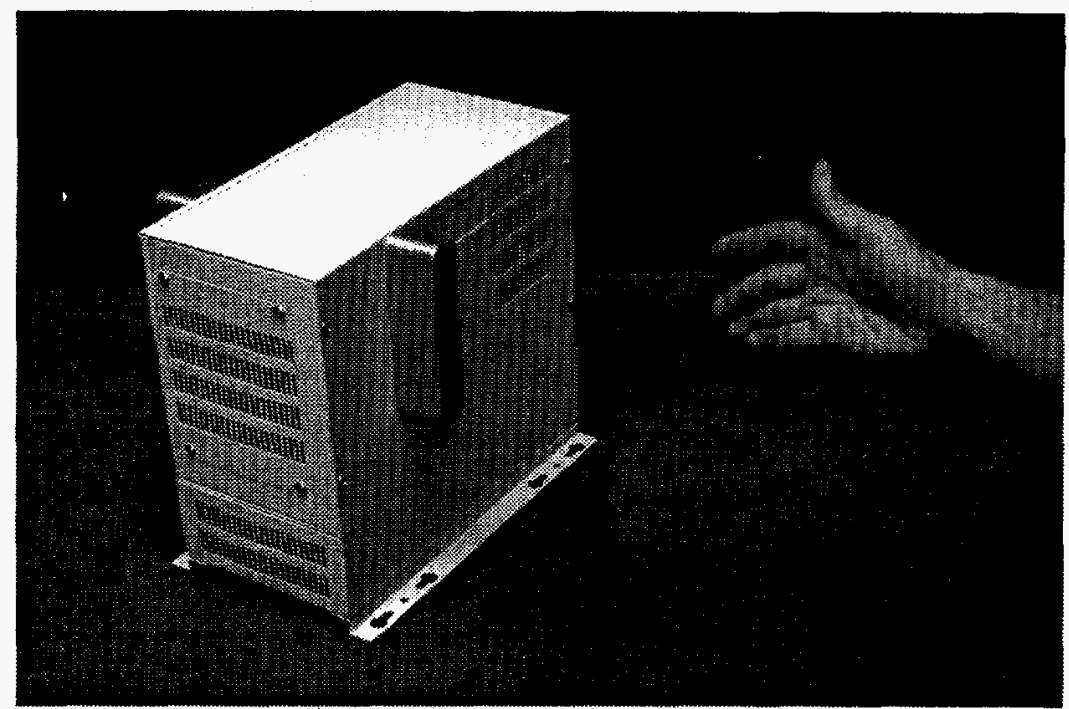

Figure 3. Scannerless Range Imager Control Computer

\section{Image Intensifier and Video Camera}

A conventional $18 \mathrm{~mm}$ GEN III image intensifier is used in the system. It provides $>50$ line pair/mm spatial resolution and $>50 \mathrm{~mA} / \mathrm{W}$ of Radiant sensitivity at the $800 \mathrm{~nm}$ operating wavelength and is well matched to the $1 / 4$ million pixels in the video camera. A relay lens is used to optically couple the image intensifier to the camera. A TI-324A camera from NEC was used in this application. This camera is a black-and-white camera using a high-resolution CCD providing 380,000 pixels (effective picture elements) on a $2 / 3$-inch format. The TI-324A CCD camera provides higher performance in resolution, signal-to-noise ratio, etc., and is more compact and lightweight than conventional CCD cameras. It has a newly developed random trigger function that was also a key attribute for use in this application.

\section{High-Speed Control Electronics}

The electronics for controlling Sandia's Scannerless Range Imager is currently configured in a 2" x 2" x 8" package. This includes a high voltage power supply to operate the image intensifier, as well as the high-speed digital and analog circuitry needed to operate the system. These electronics operate off of +5 and $+/-15$ VDC power sources, and require less that $10 \mathrm{Watts}$ of power. By transforming much of the discrete package circuitry in today's design into an application specific integrated circuit (ASIC) configuration, significant further miniaturization of these electronics is easily realizable. For the SARGE LADAR, these electronics operates at a $10 \mathrm{MHz}$ modulation frequency. This modulation frequency has demonstrated 2 inch range resolution in the laboratory. It also results in a range ambiguity interval of approximately 50 feet, which is well matched to the vehicle mobility application. 


\section{SRI Control Computer}

A low cost commercially available Single Board Computer (SBC) was used in this application. It interfaces with the video camera via a PCI based Frame Grabber. It controls the SRI instrument electronics, processes the reflectance imagery into range imagery, and provides a standardized interface from which to connect to the sensor. Reflectance and Range data from the SRI sensor is available to be transferred to the vehicle control computer through a 10 Base-T Ethernet connection. The SBC contains a PC-compatible motherboard with a $200 \mathrm{MHz}$ Intel Pentium processor with 64 Mbytes RAM. It operates under standard Microsoft Windows '95 PC operating system, and most importantly these electronics are already highly optimized for low cost, rugged, embedded industrial computer applications.

\section{Laser and Drive Electronics}

The electronics driver is configured in a 6" x 2" x 8" package. It drives a semiconductor laser diode array illuminator which has been designed for continuous duty use, $\sim 50$ foot maximum range, and wide-field ( 45 degree FOV) operation. This package includes a high frequency laser driver, and several DC-DC voltage converters that allow the electronics unit to operate from a single 24 VDC power source. The total power required for operating the laser illuminator and drive electronics is about 10 Watts.

The illumination source is a laser diode, operating in the near infrared at $804 \mathrm{~nm}$ wavelength. The optical output of the diode is a pulse train of approximately 400 -Watts peak power, $40 \mathrm{~ns}$ pulse duration, and $10 \mathrm{kHz}$ pulse repetition frequency. Average output is approximately $160 \mathrm{~mW}$. Laser output is collimated and diffused prior to exiting the laser system enclosure, so that the exit port consists of a 1-inch diameter diffuse emitter, available in a choice of either 30 or 60 degrees full cone angle.

Radiant emission per pulse is $3.8 \mu \mathrm{J} / \mathrm{cm}^{2} / \mathrm{sr}$ for a 30 -degree diffuse beam and 1.0 $\mu \mathrm{J} / \mathrm{cm}^{2} / \mathrm{sr}$ for a 60 -degree diffuse beam. Using 8.2.2.2, Table 5, and B3.2.2 of ANSI Z136.1-1993, the maximum permissible (eye-safe) exposure to

radiant emission per pulse, at a $10 \mathrm{KHz}$ prf, at $800 \mathrm{~nm}$, for a 10 -second (Class 1) exposure, is:

$$
M P E=(0.5 E-6)(10 * 10000)^{-1 / 4}(1.15 E 3) \mathrm{J} / \mathrm{cm}^{2} / \mathrm{sr}=32 \mu \mathrm{J} / \mathrm{cm}^{2} / \mathrm{sr}
$$

On this basis, the transmitter meets the emission criteria for a Class 1 (eye-safe) laser system. 


\section{EXAMPLE OF AN SRI RANGE IMAGE}

The image in Figure 4 is a typical example of the data visualization that can be accomplished with range data from the SRI. This particular data set was taken with a much more powerful laser, but at a longer range, and with a higher pixel density camera ( 1 million pixels per image). However, the rendering is only $256 \times 256$ pixels and is similar to the image quality that we are seeing with the SARGE SRI.

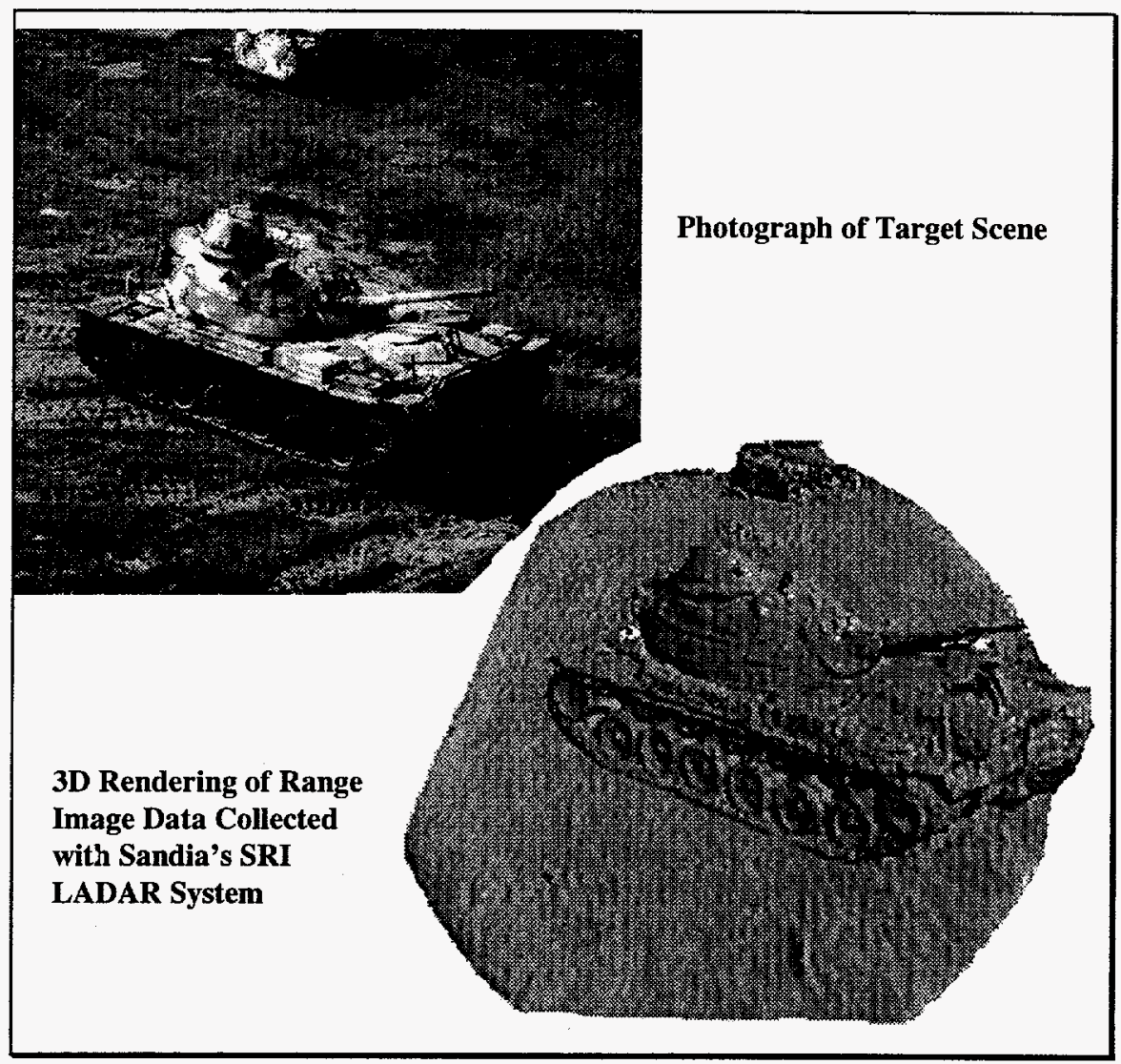

Figure 4. Example of a Range Image from the SRI Instrument

\section{AUTONOMOUS NAVIGATION}

We had intended to begin implementation and training of a neural network which would process LADAR range images for autonomous navigation in unstructured cross country environments as the final phase of this LDRD. This was recognized as a lofty goal at the outset of this work which we failed to reach. On a positive note, we did develop a useful development platform which can be used in future projects. The SARGE II robotic vehicle has all the necessary computing power and sensor interfaces required for a serious development effort in autonomous techniques for unstructured exterior environments. 
In order for the mobile platform to move safely within its environment and accomplish useful work tasks, a knowledge of the local terrain features is required. This information is necessary for identification of objects requiring manipulation as well as those requiring avoidance during movement within the workspace.

The proposed outcome of this work is to develop a mobile robotic platform that given a goal location can identify and avoid local obstacles as it traverses from its current location to the goal. This goal directed autonomous navigation scheme would use GPS to identify the robot's current coordinates in space. The path planning and path following algorithms required to reach the goal are straightforward given apriori knowledge of the clear paths and obstacles in the environment.

\section{SUMMARY}

This Lab Directed Research and Development (LDRD) project has provided support to develop a mobile robotic development platform complete with a state of the art SRI which has been custom tailored with the optimal range, FOV, and range resolution for research in autonomous navigation. This system provides Sandia with a critical capability for future research and development of autonomous navigation techniques.

The ultimate goal of this work is to develop a mobile robotic platform that can identify and avoid local obstacles as it traverses from its current location to a specified destination. This goal directed autonomous navigation scheme will use the Global Positioning System (GPS) to identify the robot's current coordinates in space and neural network processing of LADAR range images for local obstacle detection and avoidance. Contingent upon follow-on funding, future enhancements will develop neural network processing of the real-time range images provided by the SRI to traverse unstructured exterior terrain while avoiding obstacles. 


\section{REFERENCES}

M. W. Scott, Range Imaging Laser Radar, U.S. Patent 4,935,616, June 19, 1990.

Sandia National Laboratories, "Technology Transfer Opportunity - Scannerless Range Imaging System," Commerce Business Daily, September 30, 1994.

J. P. Anthes, P. Garcia, et al., "Non-scanned LADAR Imaging and Applications," Applied Laser Radar Technology, Proceedings of SPIE, Vol 1936, 1993.

P. Garcia, J. P. Anthes, et al., "Characterization of a Scannerless LADAR System," Applied Laser Radar Technology, Proceedings of SPIE, Vol 1936, 1993.

M. M. Lecavalier, et al., "Scannerless Range Imaging with a Square Wave," Guidance and Navigation - Applied Laser Radar Technology II, Proceedings of SPIE, 1995.

Ken Frazier, "Innovative Range Imager Sees How Targets Measure Up," Sandia Laboratory News, Vol. 46, No. 19, September 16, 1994.

D. H. Cress and M. M. Lecavalier, "Fusion of LADAR with SAR for Precision Strike," Proceedings from the Eighth National Symposium on Sensor Fusion, 1995.

J. Sackos, B. Bradley, B. Nellums, and C. Digert, "The Emerging Versatility of a Scannerless Range Imager," Laser Radar Technology and Applications Conference, SPIE AeroSense Symposium (Orlando, FL), paper \#2748-4, (April 1996).

J. Sackos, B. Bradley, C. Diegert, P. Ma and C. Gary, "Scannerless Terrain Mapper," SPIE International Symposium on Optical Science, Engineering, and Instrumentation, Denver, CO, August 1996.

C. Diegert, J. Sackos, and R. Nellums, "Building Accurate Geometric Models from Abundant Range Imaging Information," Laser Radar Technology and Application Conference, SPIE Aero Sense Symposium, Orlando, FL, April 1997. 